\title{
The influence of resonant MHD wave coupling in the boundary layer on the reflection and transmission process
}

\author{
J. Andries and M. Goossens \\ Centre for Plasma Astrophysics, K.U. Leuven, Celestijnenlaan 200B, 3001 Heverlee, Belgium
}

Received 25 April 2001 / Accepted 8 June 2001

\begin{abstract}
When a wave is incident on an inhomogeneous boundary layer separating two plasmas in relative motion, it is often argued that the resonant absorption rate can become negative when there is a sufficiently large velocity shear, thus giving energy back to the wave. However such treatment fails to distinguish the two energy exchange processes that are operative: extraction of wave energy by dissipative processes around the resonance point and energy exchange between the wave and the flow of the medium. By aid of the proper wave energy definitions recently discussed by Walker (2000), rather than by the conventional concept of "negative energy" waves we reveal the important dependence of the resonant amplification process on the precise structure of the boundary layer profiles. We conclude that stratification in boundary layers is very important not only because the resulting wave coupling can cause resonant instabilities for velocity shears below the Kelvin-Helmholtz threshold, but also because the coupling can stabilize the Kelvin-Helmholtz instability above this threshold.
\end{abstract}

Key words. MHD - instabilities - Sun: oscillations - Sun: magnetic fields

\section{Introduction}

Ryutova (1988) was the first to apply the concept of "negative energy" waves to resonant eigenmodes in stationary flux tubes. She showed that resonant eigenmodes become overstable if they are "negative energy" waves. However according to Hollweg et al. (1990) her work contains an inconsistency.

Hollweg et al. (1990) examined the eigenvalue problem of surface waves on a boundary layer in a plane stratified incompressible plasma (later extended to compressible but cold plasmas by Yang \& Hollweg 1991). Assuming that the boundary layer is "thin" they were able to obtain approximate analytic results. Linear profiles were used and results for different equilibrium parameters were obtained. For certain values of the velocity shear, which are beneath the threshold velocity for Kelvin-Helmholtz instability, overstability of the surface waves occurs.

Tirry et al. (1998) have investigated the same problem in a pressureless plasma. They dropped the assumption of a "thin" boundary layer and used linear profiles for the Alfvén speed but a discontinuous profile for the streaming velocity. The eigenmodes are damped when coupled to Alfvén waves with parallel longitudinal phase speed, while

Send offprint requests to: J. Andries,

e-mail: jesse.andries@wis.kuleuven.ac.be they are overstable when coupled with Alfvén waves with anti-parallel longitudinal phase speed. The overstabilities, which set in before Kelvin-Helmholtz instability, were explained by the aid of "negative energy" waves. The amount of energy absorbed by the resonant layer was shown to be negative in the case of overstability, thus giving energy to the wave rather than extracting energy from the wave.

Similar results were obtained analytically in the long wave-length approximation (equivalent to the "thin" surface approximation) by Ruderman \& Wright (1998), who investigated a model of the magnetopause with a discontinuous velocity profile. When the surface waves are "negative energy" waves they are shown to grow as a result of the dissipation at the resonance point.

Csík et al. (1998) considered the problem of wavereflection at a smooth boundary. They found that the absorption at resonances in the boundary layer could become negative thus giving energy to the wave which results in overreflection. This resonant overreflection occurred for velocity shears much lower than those needed for overreflection at a discontinuous boundary.

In Andries et al. (2000) we have studied the effect of wave resonances and of the velocity shear on the waves trapped in coronal plumes. We have considered a pressureless plasma with linear profiles for the Alfvén velocity, whereas the flow velocity is discontinuous. Similar results 
as by Tirry et al. (1998) were obtained. The threshold for resonant overstability becomes lower when the density contrast is increased.

Walker (2000) discussed the reflection and transmission at a discontinuous boundary of two counterstreaming plasmas. The conventional treatment in terms of "negative energy" waves is based on the definition of wave energy and wave energy flux so that wave energy is conserved at the boundary. An overreflected wave then requires a transmitted wave with "negative energy". According to Walker: "such an approach, while producing correct results, obscures the nature and location of the energy interchange". He discusses the proper definitions of energy density and energy flux. The location of the energy exchange is identified as the active boundary and the energy exchange is due to work done by the Reynolds and Maxwell stresses on the gradient of velocity. In this treatment there is no need to invoke "negative energy" waves.

Therefore in Andries \& Goossens (2001), where we have extended the study of Andries et al. (2000) to $\beta \neq 0$, we use Walker's approach and conclude that the resonant layer is always absorbing energy. The gained energy is due to the velocity gradients. While still using a discontinuous velocity profile we point out that these considerations make it very likely that the precise velocity profile might have an important influence on the resonant overstability. In the discontinuous model used in that paper the threshold for resonant overstability of the additional slow waves is even smaller than for the fast waves.

The aim of this paper is to show that there is indeed an important dependence of the resonant amplification process on the boundary layer profiles. We illustrate this for the driven problem and in the assumption of a "thin" boundary layer since this assumption allows us to distinguish the effects of the profiles itself from other influences.

In Sect. 2 we describe the model and the equations and discuss the solutions, and the associated wave energy flux, in the different regions of the model. The energy exchange process between a global wave (driven oscillation in the asymptotic state) and the background flow is analyzed. Using the approximation of a "thin" boundary layer, we apply these results in Sect. 3 to describe how overreflection is caused. Section 4 describes how to calculate reflection and transmission coefficients. Results are presented which clearly demonstrate the dependence of the resonant amplification process on the boundary layer profiles. Finally these results and their implications are discussed in Sect. 5 .

\section{Equations and solutions}

\subsection{Model and equations}

We use a 1-D equilibrium model consisting of two uniform regions separated by a non-uniform boundary layer $(-L<x<0)$. In the boundary layer the equilibrium quantities vary continuously from the left to the right value. The equilibrium magnetic field and the equilibrium flow $(V(x))$ are oriented in the $z$-direction. In the right medium $(x>0)$ the plasma is at rest. Because the medium is uniform in the $y$ and $z$ directions we can Fourier analyse with respect to these variables. Furthermore we are interested in the asymptotic state of the driven problem in which all perturbed quantities vary with the same frequency throughout the whole space. We thus assume solutions of the form:

$\exp \left[i\left(k_{y} y+k_{z} z-\omega t\right)\right]$.

The linearised ideal MHD equations can be reduced to the following set of two coupled ordinary differential equations for the $x$-component of the Lagrangian displacement and the Eulerian perturbation of the total pressure:

$D \frac{\mathrm{d} \xi_{x}}{\mathrm{~d} x}=C_{1} \xi_{x}-C_{2} P^{\prime}$
$D \frac{\mathrm{d} P^{\prime}}{\mathrm{d} x}=C_{3} \xi_{x}-C_{1} P^{\prime}$

In absence of gravity the coefficient functions are:

$$
\begin{aligned}
& D(x ; \omega)=\rho\left(c^{2}+v_{\mathrm{A}}^{2}\right)\left(\Omega^{2}-\omega_{\mathrm{C}}^{2}\right)\left(\Omega^{2}-\omega_{\mathrm{A}}^{2}\right) \\
& C_{1}(x ; \omega)=0 \\
& C_{2}(x ; \omega)=\left(\Omega^{2}-\omega_{\mathrm{I}}^{2}\right)\left(\Omega^{2}-\omega_{\mathrm{II}}^{2}\right) \\
& C_{3}(x ; \omega)=D \rho\left(\Omega^{2}-\omega_{\mathrm{A}}^{2}\right)
\end{aligned}
$$

with:

$$
\begin{aligned}
\omega_{\mathrm{I}, \mathrm{II}}= & \frac{1}{2}\left(k_{y}^{2}+k_{z}^{2}\right)\left(c^{2}+v_{\mathrm{A}}^{2}\right) \\
& \times\left(1 \mp\left[1-\frac{4 \omega_{\mathrm{C}}^{2}}{\left(k_{y}^{2}+k_{z}^{2}\right)\left(c^{2}+v_{\mathrm{A}}^{2}\right)}\right]^{\frac{1}{2}}\right) .
\end{aligned}
$$

The other perturbed quantities $\left(\rho_{1}, p_{1}, \ldots\right)$ can be computed once $\xi_{x}$ and $P^{\prime}$ are known. The sound speed and the Alfvén speed are defined as $c^{2}=(\gamma p) / \rho$ and $v_{\mathrm{A}}^{2}=$ $B^{2} /\left(\mu_{0} \rho\right)$ where the ratio of specific heats $\gamma=5 / 3$, as usual. The Alfvén and cusp frequency are denoted respectively by:

$$
\begin{aligned}
& \omega_{\mathrm{A}}=k_{z} v_{\mathrm{A}} \quad \text { and } \quad \omega_{\mathrm{C}}=\sqrt{c^{2} /\left(c^{2}+v_{\mathrm{A}}^{2}\right)} \omega_{\mathrm{A}} \\
& \Omega=\omega-k_{z} U(x)
\end{aligned}
$$

is the Doppler shifted frequency.

The set of ordinary differential Eqs. (1)-(2) has mobile regular singularities at the positions $x_{\mathrm{A}}$ and/or $x_{\mathrm{C}}$ where $D(x)$ vanishes:

$\omega=k_{z} U(x) \pm \omega_{\mathrm{A}}\left(x_{\mathrm{A}}\right) \quad$ and/or $\quad \omega=k_{z} U(x) \pm \omega_{\mathrm{C}}\left(x_{\mathrm{C}}\right)$. $(3)$

As both $\omega_{\mathrm{A}}(x)$ and $\omega_{\mathrm{C}}(x)$ are functions of $x$, they define two continuous ranges of frequencies referred to as the Alfvén continuum and the slow or cusp continuum respectively. These continua are Doppler shifted due to the presence of the flow as indicated by Eq. (3). For positive (negative) frequencies we call a resonance according to the $+(-)$ sign a forward resonance and according to the $-(+)$ sign a backward resonance. This corresponds to the coupling with Alfvén or slow waves of parallel or anti-parallel longitudinal phase speed. 


\subsection{Uniform regions}

By substitution of Eq. (1) into Eq. (2) one second order differential equation in $P^{\prime}$ can be derived. In the uniform regions this becomes:

$\frac{\mathrm{d}^{2} P^{\prime}}{\mathrm{d} x^{2}}=\kappa_{x}^{2} P^{\prime}$

with:

$\kappa_{x}^{2}=-\frac{C_{2} C_{3}}{D^{2}}=-\frac{\left(\Omega^{2}-\omega_{\mathrm{I}}^{2}\right)\left(\Omega^{2}-\omega_{\mathrm{II}}^{2}\right)}{\left(c^{2}+v_{\mathrm{A}}^{2}\right)\left(\Omega^{2}-\omega_{\mathrm{C}}^{2}\right)}$

and thus yields solutions:

$P^{\prime} \sim \exp \left(\kappa_{x} x\right)$

We are studying a wave that is incident on the boundary from the right medium (subscript $\mathrm{r}$ ). Part of this wave is reflected and part of it is transmitted in the left medium (subscript 1). Thus we study frequencies for which $\kappa_{x \mathrm{r}}^{2}<0$ so that we have propagating waves in the right medium. The solution in the right medium is then composed of an incident and a reflected wave:

$P_{\mathrm{r}}^{\prime}=A_{\mathrm{i}} \exp \left(\kappa_{x \mathrm{r}} x\right)+A_{\mathrm{r}} \exp \left(-\kappa_{x \mathrm{r}} x\right)$

The root $\kappa_{x \mathrm{r}}$ has to be chosen correctly to assure that the first part is an incoming wave and the second part is an outgoing wave. In the left medium the solution:

$P_{1}^{\prime}=A_{\mathrm{t}} \exp \left(\kappa_{x \mathrm{l}} x\right)$

has to represent a wave propagating to the left when $\kappa_{x \mathrm{l}}^{2}<0$ or vanishing at infinity when $\kappa_{x \mathrm{l}}^{2}>0$.

Csík et al. (1998) considered only frequencies with vanishing wave amplitude in the left medium, thus eliminating the possibility of transmission. However there is no difficulty in calculating the reflection coefficients if the wave is partially transmitted. A problem in their paper was the interpretation of this partial transmission. In the absence of transmission they straightforwardly interpreted the part of the energy that is not reflected as the energy absorbed by the resonance. Then if the wave is overreflected the resonance is giving energy to the wave. However this causes problems of interpretation when we have to deal with partially transmitted waves since it is not clear whether the "lost" energy is transmitted or absorbed.

We show that by using the wave energy flux definitions discussed by Walker (2000) the solution to these problems is clear and reveals the more detailed structure of the energy transfer in the boundary layer. We therefore prefer to use Walker's definition of energy flux to determine the direction of the wave propagation rather than using the phase velocity and the considerations about its relationship to the group velocity made by Csík et al. (1998), although in practice the results are equivalent. The $x$-component of the wave energy flux (averaged over one cycle) is computed as (Walker 2000; Adam 1978):

$F_{x}=\frac{1}{2} \Omega \operatorname{Re}\left(-i \xi_{x} \overline{P^{\prime}}\right)$ where the horizontal bar denotes the complex conjugate. Since the other components of the energy flux are not relevant to the present discussion, we denote $F_{x}$ as $F$. The energy flux in the left medium becomes:

$F_{1}=\frac{\operatorname{Im}\left(\kappa_{x \mathrm{l}}\right)}{2} \frac{\Omega}{\rho\left(\Omega^{2}-\omega_{\mathrm{Al}}^{2}\right)}\left|A_{\mathrm{t}}\right|^{2}$.

Thus we have to choose the root $\kappa_{x l}$ so that:

$\left(\operatorname{Im}\left(\kappa_{x \mathrm{l}}\right) \frac{\Omega}{\Omega^{2}-\omega_{\mathrm{Al}}^{2}}\right)<0$.

This choice depends on whether the wave is fast or slow but also on $\Omega$ itself since $\Omega$ can become negative due to the Dopplershift although $\omega$ is positive. For the right medium we get:

$F_{\mathrm{r}}=\frac{\operatorname{Im}\left(\kappa_{x \mathrm{r}}\right)}{2} \frac{\omega}{\rho\left(\omega^{2}-\omega_{\mathrm{Ar}}^{2}\right)}\left(\left|A_{\mathrm{r}}\right|^{2}-\left|A_{\mathrm{i}}\right|^{2}\right)$.

This clearly shows that the energy flux consists of one part carried in by the incident wave and one part carried out by the reflected wave. Now the choice of the root has to be taken so that the factor in front is positive:

$\left(\operatorname{Im}\left(\kappa_{x \mathrm{r}}\right) \frac{\omega}{\omega^{2}-\omega_{\mathrm{Ar}}^{2}}\right)>0$.

Also note that for both the left and the right solution the wave indeed does not carry any energy flux when $\kappa_{x}^{2}>0$.

\subsection{The boundary layer}

In the boundary layer the set of Eqs. (1), (2) can be integrated numerically. However in the vicinity of a resonance this method fails because of the singularity. This is just a result from the fact that ideal MHD breaks down around the resonance point. Due to the large gradients dissipation becomes important. Sakurai et al. (1991) have included dissipative effects in the resonance layer to remove the singularity and derived connection formulae to connect the solutions to the right and the left of the resonance layer. This can be done because of the very large Reynolds numbers that make sure the resonance layer is very thin (Goossens et al. 1992; Goossens et al. 1995). However these dissipative effects are not essential to the physical result of absorption or amplification of a wave at the resonance. In the ideal initial-value problem the correct connection formulae are obtained by the Landau prescription for going around the pole (Hollweg et al. 1990; Sedlacek 1971). Whether the extracted energy is dissipated into heat or just stored in an infinitely large energy reservoir at the position of the resonance is mathematically unimportant. The important thing is that energy is extracted and thus an energy flux is created.

The jump relations for $\xi_{x}$ and $P^{\prime}$ at the Alfvén resonance are (e.g. Goossens et al. 1992):

$\left[P^{\prime}\right]=0$
$\left[\xi_{x}\right]=-i \pi \operatorname{sign}(\Omega) \frac{k_{y}^{2} P^{\prime}}{\rho|\Delta|}$. 
Here $\Delta=\mathrm{d} / \mathrm{d} x\left(\Omega^{2}-\omega_{\mathrm{A}}^{2}\right)$ and all the quantities are evaluated at the resonant position. From Eq. (4) it can be seen that a wave carries energy only when $P^{\prime}$ and $\xi_{x}$ are out of phase. A resonance therefore creates an energy flux since the jump conditions prohibit that $P^{\prime}$ and $\xi_{x}$ are in phase on both sides of the resonant layer. The jump in energy flux over the resonance layer can be found immediately from the jump conditions (the resonant layer is normally very thin compared to the length scales of the differences in the flow velocity so that we can assume $\Omega$ to be constant across the resonant layer):

$[F]=-\frac{\pi|\Omega| k_{y}^{2}}{2 \rho|\Delta|}\left|P^{\prime}\right|^{2}$.

Thus energy is always absorbed by the resonance. For the cusp resonant layer similar results can be obtained. This result is very logical since the dissipative effects are converting magnetic and kinetic energy into heat. However it is in contradiction to the explanation of resonant overreflection by Csík et al. (1998) as negative absorption by the resonance. Obviously, the gained energy is coming out of the flow, but this calculation makes it even more clear that the energy exchange process does not take place at the resonant position.

Therefore let us derive in general the change of energy flux (away from the resonances):

$$
\begin{aligned}
2 \frac{\mathrm{d}(F)}{\mathrm{d} x} & =\frac{\mathrm{d}(\Omega)}{\mathrm{d} x} \operatorname{Re}\left(-i \xi_{x} \overline{P^{\prime}}\right)+\Omega \frac{\mathrm{d}\left(\operatorname{Re}\left(-i \xi_{x} \overline{P^{\prime}}\right)\right)}{\mathrm{d} x} \\
& =-k_{z} \frac{\mathrm{d}(V)}{\mathrm{d} x} \operatorname{Re}\left(-i \xi_{x} \overline{P^{\prime}}\right)+\Omega \frac{\mathrm{d}\left(\operatorname{Re}\left(-i \xi_{x} \overline{P^{\prime}}\right)\right)}{\mathrm{d} x} \\
\frac{\mathrm{d}(F)}{\mathrm{d} x} & =-k_{z} \frac{\mathrm{d}(V)}{\mathrm{d} x} \frac{F}{\Omega}+\Omega \frac{\mathrm{d}\left(\frac{F}{\Omega}\right)}{\mathrm{d} x} .
\end{aligned}
$$

Since the averaged energy fluxes in the $y$ and $z$ directions are clearly constant with respect to $y$ and $z$ respectively, the result is actually the divergence of the energy flux or thus the work done on the wave. There are clearly two contributions: one associated with the velocity gradients and one associated with the variation of the perturbed quantities. At first sight this division may seem artificial since the second term also possesses changes indirectly due to the velocity gradients. However, from Eqs. (1), (2):

$$
\begin{aligned}
\frac{\mathrm{d}\left(\frac{F}{\Omega}\right)}{\mathrm{d} x} & =\frac{\mathrm{d}\left(\operatorname{Re}\left(-i \xi_{x} \overline{P^{\prime}}\right)\right)}{\mathrm{d} x} \\
& =\operatorname{Re}\left[-i\left(\frac{\mathrm{d} \xi_{x}}{\mathrm{~d} x} \overline{P^{\prime}}+\xi_{x} \frac{\overline{\mathrm{d} P^{\prime}}}{\mathrm{d} x}\right)\right] \\
& =\operatorname{Re}\left[-i\left(-\frac{C_{2}}{D}\left|P^{\prime}\right|^{2}+\frac{C_{3}}{D}\left|\xi_{x}\right|^{2}\right)\right] \\
& =0 .
\end{aligned}
$$

Thus the second term in (8) vanishes (except at resonances where $D=0$ and thus the above derivation is invalid). The resulting formula (8) then eventually tells us the same. If we compare the result (8) with Eq. (4.15) in Walker (2000) we conclude that:

$\frac{\kappa_{z} F}{\Omega}=T_{x, z}$
The conserved quantity is actually the $(x, z)$ component of the stress tensor (averaged over one cycle). The variations in $\xi_{x}$ and $P^{\prime}$ are due to the exchange of energy between the magnetic field disturbances and the pressure disturbances, between the Maxwell stresses and the Reynolds stresses. Obviously, this happens in such a way that the averaged stresses remain balanced. Consequently wave energy is conserved as long as velocity gradients are absent or the averaged stresses are zero. The change of the energy flux is proportional to the change in Dopplershifted frequency (velocity field) and proportional to the averaged $(x, z)$ component of the stress tensor. This clarifies how the resonances cause non zero $(x, z)$ stresses at at least one side of the resonance point and thereby enable the exchange of energy between the flow and the wave.

\section{A simple energy flux explanation of over-reflection}

First of all we can apply the findings of the previous section to explain overreflection for a discontinuous boundary. In that case the effect of the resonances in the boundary layer is neglected. Since the influence of the resonances is neglected $T_{x, z}$ is constant throughout space. If there is no transmission possible $T_{x, z}=0$ in the left region. Thus, the energy flux is zero everywhere, including in the righthand region where the amplitude of the reflected wave is therefore equal (in magnitude) to the amplitude of the incoming wave. Nor absorption nor overreflection occur here. On the other hand suppose there is transmission. In that case the energy flux in the left medium is negative. The sign of the energy flux in the right medium is determined by the signs of the right and left hand Dopplershifted frequencies. Overreflection occurs if and only if:

$\frac{\omega}{\Omega_{1}}<0$

This can only be satisfied for sufficiently large velocity shear. The same relationship was derived in Andries \& Goossens (2001) for (non-resonant) Kelvin-Helmholtz instability of trapped body modes in a plume, and it is equivalent to the condition for the appearance of "negative energy" waves.

Notice that the final asymptotic state is reached through the balancing of the stresses. When no transmission is possible the driver is unable to create any resulting stresses in the left region. Thus in the asymptotic state there are no stresses whatsoever. The driving stresses are counteracted by the stresses that are reflected. When transmission is possible, the driver is able to produce stresses that are well defined by the equilibrium parameters in the left region. Although the disturbances are driven in the right medium the asymptotic state is eventually defined by the equilibrium parameters of the left region (and the driving frequency off course).

If resonances are included, the problem gets more complicated since $T_{x, z}$ can change at the resonant positions. 
Moreover these variations are proportional to $\left|P^{\prime}\right|^{2}$ which itself varies. In order to overcome this last complication we assume that the boundary layer is "thin". The assumption of a "thin" surface was also made by Hollweg et al. (1990). They argue that when the surface or boundary layer is "thin" $P^{\prime}$ remains approximately constant across the boundary layer. In saying so they actually define what they mean by a "thin" surface. Furthermore under that assumption they also neglect some contributions to the change of $\xi_{x}$ across the boundary layer. Indeed, we call the boundary layer "thin" if it is smaller than the lengthscales on which the perturbed quantities vary. Under that assumption $\xi_{x}$ and $P^{\prime}$ are approximately constant across the boundary layer. However if there is a resonance present the lengthscales of variation of $\xi_{x}$ and $P^{\prime}$ become very small near the resonance point. Thus the variations in these quantities inside the resonance layer can not be neglected. These variations are given by the jump conditions and correspond exactly to the term in the variation of $\xi_{x}$ that is kept by Hollweg et al. (1990). Outside the resonant layers the variations of the perturbed quantities are neglected.

Assume that the conditions are so that transmission is not possible and that two resonances occur: a forward and a backward Alfvén resonance (therefore $\beta=0$ ). The backward resonance occurs further to the left than the forward resonance. This becomes clear in the next section, where we look at different profiles and show that no backward resonances can be present without a forward resonance contrary to what is suggested in Csík et al. (1998), Tirry et al. (1998), Andries et al. (2000) and Andries \& Goossens (2001).

Because of the assumption of no transmission the energy flux in the left medium is zero, $F_{1}=0$. In spite of the velocity gradients the energy flux is also zero at the left side of the backward resonance region, since $T_{x, z}=0$. Then we apply the energy flux jump relation (7) to obtain the energy flux at the right side of the backward resonance (subscript b means at the position of the backward resonance, $P_{0}^{\prime}$ is the pressure perturbation which is constant throughout the entire boundary layer):

$F_{\mathrm{br}}=-\frac{\pi\left|\Omega_{\mathrm{b}}\right| k_{y}^{2}\left|P_{0}^{\prime}\right|^{2}}{2 \rho_{\mathrm{b}}\left|\Delta_{\mathrm{b}}\right|}$

which is clearly negative, because the resonance is extracting energy from the incoming wave (from the right). However because the energy flux and thus $T_{x, z}$ are nonzero the velocity gradients supply energy so that at the left side of the forward resonance the energy flux becomes (subscript $\mathrm{f}$ means at the position of the forward resonance):

$$
\begin{aligned}
F_{\mathrm{fl}} & =\frac{\Omega_{\mathrm{f}}}{\Omega_{\mathrm{b}}} F_{\mathrm{br}} \\
& =-\Omega_{\mathrm{f}} \frac{\pi \operatorname{sign}\left(\Omega_{\mathrm{b}}\right) k_{y}^{2}\left|P^{\prime}\right|^{2}}{2 \rho_{\mathrm{b}}\left|\Delta_{\mathrm{b}}\right|} .
\end{aligned}
$$

This is positive ( $\Omega_{\mathrm{f}}$ and $\Omega_{\mathrm{b}}$ have opposite signs) and thus the flow has provided the energy required to change the negative energy flux into a positive energy flux. We now again apply the jump conditions (7) over the forward resonance to get the energy flux at the right side of the forward resonance:

$F_{\mathrm{fr}}=-\Omega_{\mathrm{f}} \frac{\pi \operatorname{sign}\left(\Omega_{\mathrm{b}}\right) k_{y}^{2}\left|P^{\prime}{ }_{0}\right|^{2}}{2 \rho_{\mathrm{b}}\left|\Delta_{\mathrm{b}}\right|}-\frac{\pi\left|\Omega_{\mathrm{f}}\right| k_{y}^{2}\left|P^{\prime}{ }_{0}\right|^{2}}{2 \rho_{\mathrm{f}}\left|\Delta_{\mathrm{f}}\right|}$.

Thus part of the energy flux is absorbed by the resonance layer. The sign of the resulting energy flux depends on the relative effect of the two resonances. We can then find the resulting energy flux at the right side of the boundary layer as:

$$
\begin{aligned}
F_{\mathrm{r}} & =\frac{\omega}{\Omega_{\mathrm{f}}} F_{\mathrm{fr}} \\
& =-\frac{\omega \pi k_{y}^{2}\left|P^{\prime}{ }_{0}\right|^{2}}{2}\left[\frac{\operatorname{sign}\left(\Omega_{\mathrm{b}}\right)}{\rho_{\mathrm{b}}\left|\Delta_{\mathrm{b}}\right|}+\frac{\operatorname{sign}\left(\Omega_{\mathrm{f}}\right)}{\rho_{\mathrm{f}}\left|\Delta_{\mathrm{f}}\right|}\right] \\
& =-\frac{|\omega| \pi k_{y}^{2}\left|P^{\prime}{ }_{0}\right|^{2}}{2}\left[\frac{\operatorname{sign}\left(\Omega_{\mathrm{b}} / \omega\right)}{\rho_{\mathrm{b}}\left|\Delta_{\mathrm{b}}\right|}+\frac{\operatorname{sign}\left(\Omega_{\mathrm{f}} / \omega\right)}{\rho_{\mathrm{f}}\left|\Delta_{\mathrm{f}}\right|}\right] .
\end{aligned}
$$

Thus it is clear that resonances provide separate contributions and that the contribution of a backward resonance is always positive whereas that of a forward resonance is always negative. The reason is that the difference in Dopplershifted frequency between the resonant position and the right side of the boundary layer determines whether the negative flux is changed into a positive one by the velocity gradients or not. Since the sign of the Dopplershifted frequency at a backward resonance is opposite to the sign of the frequency enough energy is extracted out of the flow to provide the energy extracted at the resonance. In case of a forward resonance this is not true and the net effect is extraction of energy out of the wave. If the computed energy flux (10) is positive then there is more energy reflected at the boundary than there is incident on the boundary and thus we have overreflection.

This calculation shows that resonant overreflection only occurs if the contribution of the backward resonance is larger than that of the forward resonance. This depends on several parameters on the resonant positions and thus on the profiles in the boundary layer. We want to emphasize that there is in principle no problem in treating boundary layers that are not "thin". The set of Eqs. (1)-(2) can be integrated numerically in the boundary layer. The reason to confine our interest to "thin" surfaces is that we can focus on the effects of the profiles itself on the two (or more) contributions in (10). If the boundary layer is not thin $P^{\prime}$ varies through the boundary and affects the relative effect of the two contributions as well. It is then hard to distinguish the effects of the changing amplitude and of the changing equilibrium quantities in the boundary.

\section{Computation of the reflection coefficient}

\subsection{Method}

We only look at the situation $\beta=0$. There are several reasons for doing so, while the treatment of $\beta \neq 0$ is not 
essentially more difficult. First of all the assumption is made to facilitate the interpretation of the results. When studying fast incident waves the interesting situations with backward resonances would involve at least 3 or 4 resonances when $\beta \neq 0$. This would make interpretation very hard because more terms have to be compared. The results would become even more dependent on the profiles because there are more parameters defining the profiles ( $\beta$ determines the relationship between the Alfvén speed profile and the slow speed profile). Second, there is a problem in analyzing slow waves in the "thin" boundary approximation. The slow waves behave very an-isotropically and propagate mainly in the direction of the magnetic field and thus have small perpendicular wavelengths. In the computation some finite thickness $L$ for the boundary layer has to be used. The assumption of a thin surface is then satisfied by taking an imposed wavenumber $k=\sqrt{k_{z}^{2}+k_{y}^{2}} \ll 1 / L . \kappa_{x}$ is of the order of $k$ or smaller except for slow body waves $\left(\omega_{\mathrm{C}}<\omega<\omega_{\mathrm{I}}\right)$ where it turns out that $\kappa_{x}$ becomes very large (infinite) for $\omega \rightarrow \omega_{\mathrm{C}}$. Thus for all except slow body waves this choice of $k$ assures large perpendicular wavelengths. In short, putting $\beta=0$ facilitates the treatment and it is sufficient for our purpose: i.e. showing that the profile of the boundary layer has a strong effect on the modifications made by resonant behaviour to the process of reflection and transmission.

For the computation of the reflection coefficient a similar treatment as in Sect. 3 is followed. The amplitude reflection and transmission coefficients are defined as:

$R=\frac{A_{\mathrm{r}}}{A_{\mathrm{i}}} \quad$ and $\quad T=\frac{A_{\mathrm{t}} \exp \left(-\kappa_{x \mathrm{l}} L\right)}{A_{\mathrm{i}}}$.

Some fixed value for the pressure perturbation in the boundary layer $P_{0}^{\prime}$ can be assumed. Equating the right and left solutions for $P^{\prime}$ provides a first equation:

$T=R-1$.

Furthermore $\xi_{x}$ has to be continuous (except at the resonances). At the left side of the boundary we have:

$\xi_{x \mathrm{l}}=\frac{\kappa_{x \mathrm{l}}}{\rho_{\mathrm{l}}\left(\Omega_{\mathrm{l}}^{2}-\omega_{\mathrm{Al}}^{2}\right)} A_{\mathrm{t}} \exp \left(-\kappa_{x \mathrm{l}} L\right)=D_{\mathrm{l}} A_{\mathrm{t}} \exp \left(-\kappa_{x \mathrm{l}} L\right)$.

At the right side of the boundary layer we then get:

$$
\begin{aligned}
\xi_{x \mathrm{r}} & =D_{\mathrm{l}} A_{\mathrm{t}} \exp \left(-\kappa_{x \mathrm{l}} L\right)-\sum_{i} \frac{i \pi \operatorname{sign}\left(\Omega_{i}\right) k_{y}^{2} P_{0}^{\prime}}{\rho_{i}\left|\Delta_{i}\right|} \\
& =D_{\mathrm{l}} A_{\mathrm{t}} \exp \left(-\kappa_{x \mathrm{l}} L\right)-\sum_{i} i \pi k_{y}^{2} \frac{\operatorname{sign}\left(\Omega_{i}\right)}{\rho_{i}\left|\Delta_{i}\right|} A_{\mathrm{t}} \exp \left(-\kappa_{x \mathrm{l}} L\right) \\
& =\left(D_{\mathrm{l}}+D_{0}\right) A_{\mathrm{t}} \exp \left(-\kappa_{x \mathrm{l}} L\right)=D A_{\mathrm{t}} \exp \left(-\kappa_{x \mathrm{l}} L\right)
\end{aligned}
$$

where the sum is taken over all resonances in the boundary layer. On the other hand in the right medium we have:

$\xi_{x \mathrm{r}}=\frac{\kappa_{x \mathrm{r}}}{\rho_{\mathrm{r}}\left(\omega^{2}-\omega_{\mathrm{Ar}}^{2}\right)}\left(A_{\mathrm{r}}+A_{\mathrm{i}}\right)=D_{\mathrm{r}}\left(A_{\mathrm{r}}+A_{\mathrm{i}}\right)$.

Equating (12) and (13) provides a second equation:

$D T=D_{\mathrm{r}}(R+1)$.
The solutions of (11) and (14) are:

$R=\frac{D+D_{\mathrm{r}}}{D-D_{\mathrm{r}}} \quad$ and $\quad T=\frac{2 D_{\mathrm{r}}}{D+D_{\mathrm{r}}}$.

However we are more interested in the energy reflection and transmission coefficients, simply defined by the comparison of the reflected and transmitted energy with the incident flux. From (5) and (6) we clearly have:

$\mathcal{R}=\frac{\left|A_{\mathrm{r}}\right|^{2}}{\left|A_{\mathrm{i}}\right|^{2}}=R \bar{R}=|R|^{2}$

$\mathcal{T}=-\frac{\Omega_{1}}{\omega} \frac{\operatorname{Im} D_{1}}{\operatorname{Im} D_{\mathrm{r}}} \frac{\left|A_{\mathrm{t}}\right|^{2}}{\left|A_{\mathrm{i}}\right|^{2}}=-\frac{\Omega_{1}}{\omega} \frac{\operatorname{Im} D_{\mathrm{l}}}{\operatorname{Im} D_{\mathrm{r}}}|T|^{2}$.

These expression and those for the amplitude reflection and transmission coefficients can be shown to be equivalent to those obtained by Walker (2000) when $D_{0}=0$. If the amplitude reflection coefficient is larger than 1 (in magnitude) overreflection occurs. Thus let us first take a closer look at formula (15) for the reflection coefficient. Since $D_{\mathrm{r}}$ is always imaginary (we consider an incoming wave) the real part of the denominator and the numerator are the same and we clearly have:

$|R|>1 \Longleftrightarrow\left|\operatorname{Im}\left(D+D_{\mathrm{r}}\right)\right|>\left|\operatorname{Im}\left(D-D_{\mathrm{r}}\right)\right| \Longleftrightarrow$

$\operatorname{sign}(\operatorname{Im} D)=\operatorname{sign}\left(\operatorname{Im} D_{\mathrm{r}}\right) \Longleftrightarrow \operatorname{sign}(\operatorname{Im} D)=\operatorname{sign}(\omega)$.

The last step is a consequence of the choice of the root $\kappa_{x \mathrm{r}}$. When no transmission is possible $\kappa_{x 1}$ and therefore $D_{1}$ is real. Then the only imaginary contributions to $D$ are the contributions of the resonances $D_{0}$ and the condition for overreflection becomes:

$\sum_{i} \frac{\operatorname{sign}\left(\Omega_{i} / \omega\right)}{\rho_{i}\left|\Delta_{i}\right|}<0$.

We thus again retrieve the result of Sect. 3. On the other hand if no resonances occur and no transmission is possible the reflection coefficient just represents a phase shift of the reflected wave with respect to the incoming wave since $|R|=1$ because $D=D_{1}$ is real and $D_{\mathrm{r}}$ imaginary. If no resonances occur but there is transmission the choice of the root $\kappa_{x 1}$ is so that $\left.\operatorname{sign}\left(\operatorname{Im} D_{1}\right)\right)=-\operatorname{sign}\left(\Omega_{1}\right)$. And thus the condition for overreflection (9) is again retrieved.

\subsection{Results}

In the presentation of the results, velocity is scaled with respect to the right Alfvén speed. Some different profiles are visualized in Figs. 1 to 7 . The horizontal line indicates the $z$-component of the phase speed $\omega / k_{z}$ of the imposed incoming wave. The other lines show the profiles of Dopplershifted Alfvén speed $\pm v_{\mathrm{A}}+V$ (full line) and the Dopplershifted cut-off speed $\pm \omega_{\mathrm{II}} / k_{z}+V$ (dashed line), which indicates the distinction between propagating and evanescent waves. Thus resonances are marked by the intersections between the horizontal line and the 


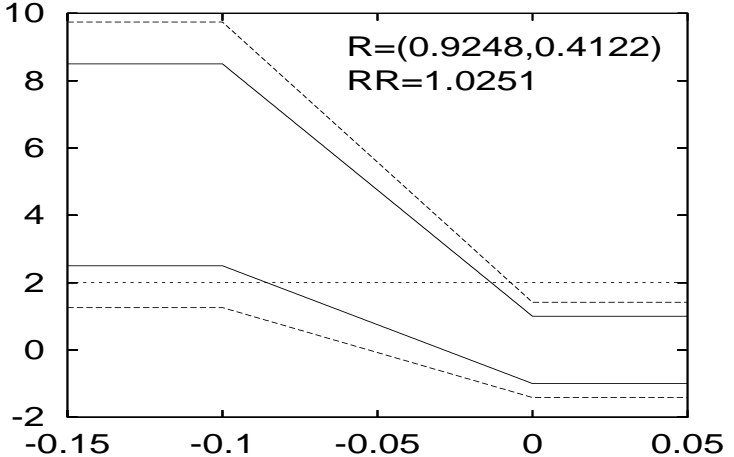

Fig. 1. Possible profiles for Alfvén speed $\pm v_{\mathrm{A}}+V$ (full line) and cut-off speed $\pm \omega_{\text {II }} / k_{z}+V$ in a boundary layer of thickness 0.1 separating the incoming wave with wavenumbers $k_{z}=1 / \sqrt{2}=$ $k_{y}$ and longitudinal phase speed $\omega / k_{z}=2$ from a region with $v_{\mathrm{Al}}=3$ and $V=5.5 . R$ and $R R$ indicate the amplitude and energy reflection coefficients.

full lines. Intersections with the upper full lines correspond to forward resonances, while intersections with the lower full line are backward resonances. Since the horizontal line lies above the upper dashed line in the right medium it first has to intersect the upper full line before it can intersect the lower full line. Thus backward resonances are impossible without forward resonances. Also in Csík et al. (1998), Tirry et al. (1998), Andries et al. (2000) and Andries \& Goossens (2001) where backward resonances are presented without forward resonances, forward resonances occur, but they occur at the discontinuity and have no effect. This can be seen by rewriting $|\Delta|$ as follows:

$$
|\Delta|=2 v_{\mathrm{A}} k_{z}^{2}\left|\frac{\mathrm{d}\left(V \pm v_{\mathrm{A}}\right)}{\mathrm{d} x}\right| .
$$

The \pm sign corresponds to forward or backward resonances respectively. Thus the larger the derivative at the resonance point the smaller the contribution in (10). For resonances occurring at a discontinuity the contribution becomes zero. Therefore the results obtained with discontinuous profiles are valid and consistent with the present treatment of continuous profiles in the sense that the discontinuous case can be understood as a limiting case of the continuous case.

We first present solutions for a model in which the wave is incident from a medium with higher density and unable to be transmitted through the boundary. The parameters are set as follows: $k_{z}=1 / \sqrt{2}=k_{y}, L=0.1, \omega / k_{z}=2$, $v_{\mathrm{Al}}=3$ and $V=5.5$ (Figs. 1,2 ) or $V=4.6$ (Fig. 3). Figure 1 shows a profile in which the velocity and Alfvén speed change linearly over the entire boundary layer. A forward and a backward resonance occur. The effect of the backward resonance dominates because the density as well as the derivative of the Dopplershifted Alfvén profile are smaller at the backward resonance than at the forward resonance. Thus as indicated, this situation leads to overreflection.

Now let us look at the profiles in Fig. 2. They are still piecewise linear profiles resulting from linear profiles of the
Alfvén speed and of the velocity. However the region where the Alfvén speed varies is located more to the right than the region where the velocity changes. Moreover the thickness of these regions is different in the different pictures. Figure 2a shows a profile where the Alfvén speed changes over a broad distance while the velocity field changes more abruptly. This situation does not lead to overreflection. The fact that the derivative of the Alfvén profile is much smaller at the forward resonance seems to overcome the larger density at the point resulting in the dominance of the forward resonance. In Fig. $2 \mathrm{~b}$ the region where the velocity changes is broader, while the Alfvén speed changes abruptly. This results in a small derivative at the backward resonance, thereby dominating and causing overreflection. In Fig. 2c an intermediate region is introduced between the left region where the velocity changes and the right region where the Alfvén speed changes. Again the smaller derivative makes the forward resonance dominate. Eventually Fig. 2d shows a situation in which there is an overlap of the two boundary layers, apparently resulting in overreflection.

We now turn to Fig. 3. In these figures the transition region for the velocity is located on the right and the transition region for the Alfvén speed on the left. This results in a strange behaviour of the backward profile which has a maximum in the boundary layer. If we would have kept all the parameters as in Fig. 2, the third resonance would be absent. In that case the results would be clear (at least for situations a, b, c) and not too exciting: because at both resonances the derivatives are equal and the density is equal, both contributions annihilate each other, thus leading to pure reflection. However we have considered somewhat slower flow speeds to illustrate the situations where the third resonance is present. Since the two first resonances annihilate each other the third resonance, which is a backward one, dominates resulting in overreflection in all cases.

Now let us take a look at Figs. 4 to 6 . In these cases the wave is incident from a medium with lower density. The parameters are $k_{z}=1 / \sqrt{2}=k_{y}, L=0.1, \omega / k_{z}=2$, $v_{\mathrm{Al}}=\frac{1}{3}$ and $V=2.4$. It is unnecessary to discuss all of these figures in detail since they are easily interpreted. The conclusion is that the different profiles of the boundary layer that are shown all separate the same two homogeneous regions but the calculated reflection coefficients differ strongly depending on the boundary layer profiles.

Until now we have restricted our attention to cases in which the resonant overreflection mechanism is the only overreflection mechanism operative. However we would also like to know whether the resonant effects can change the KH-type overreflection significantly or not. The answer is yes, which can be seen immediatelly from Fig. 7. The situation is essentially the same as in Fig. 2a. Although, the parameters are slightly different (this is explained below). The velocity shear is chosen large enough to have wave transmission. If the resonances are neglected (the boundary is taken to be discontinuous) this results in overreflection $(\mathcal{R}=12.4187, \mathcal{T}=30.8727)$. But as can be 
a

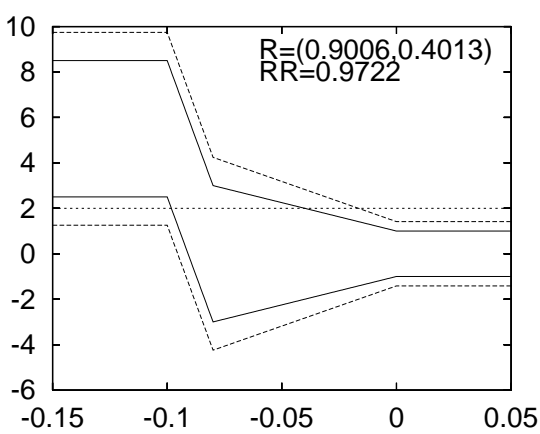

C

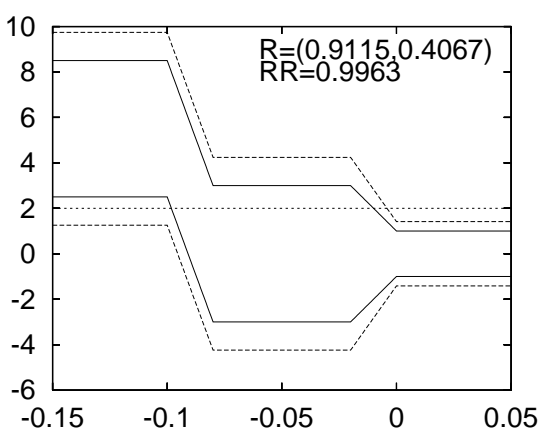

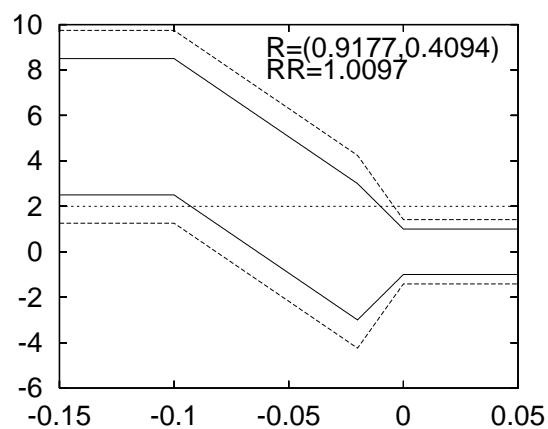

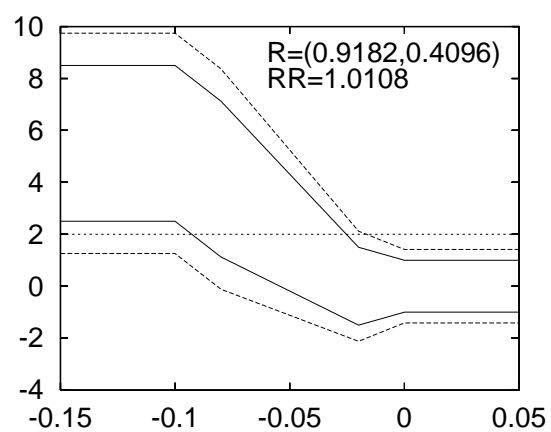

Fig. 2. Different possible profiles for Alfvén speed $\pm v_{\mathrm{A}}+V$ (full line) and cut-off speed $\pm \omega_{\mathrm{II}} / k_{z}+V$ in a boundary layer of thickness 0.1 separating the incoming wave with wavenumbers $k_{z}=1 / \sqrt{2}=k_{y}$ and longitudinal phase speed $\omega / k_{z}=2$ from a region with $v_{\mathrm{Al}}=3$ and $V=5.5 . R$ and $R R$ indicate the amplitude and energy reflection coefficients.
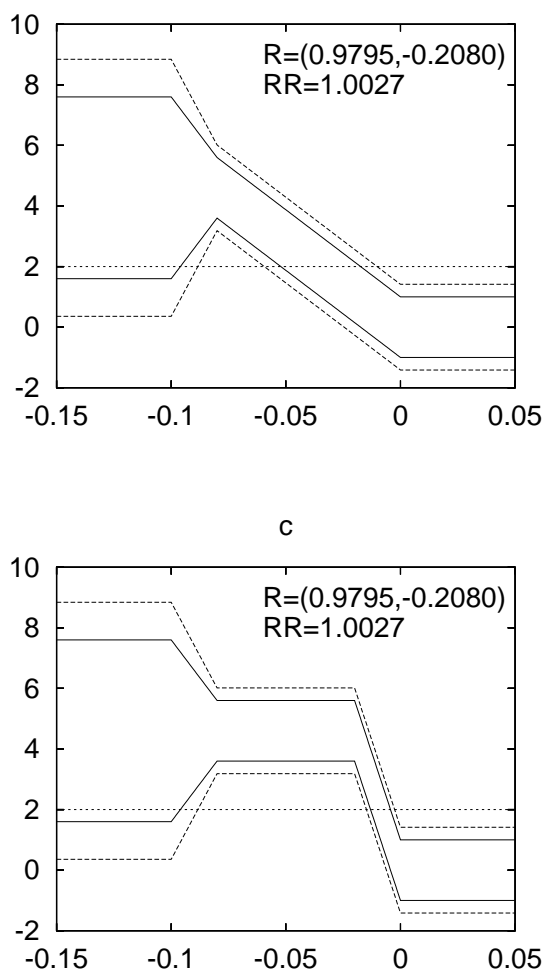

b

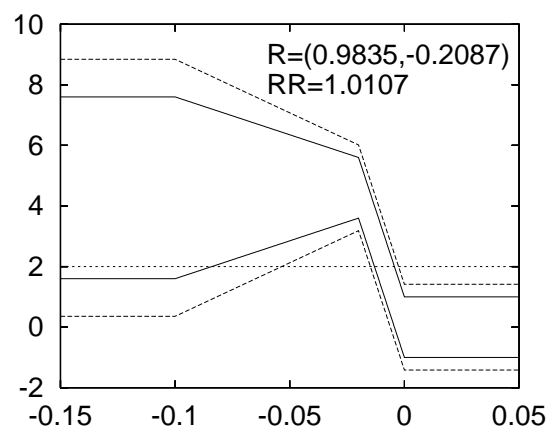

d

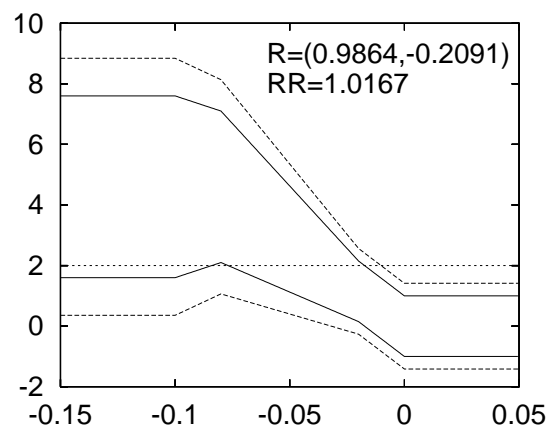

Fig. 3. Different possible profiles for Alfvén speed $\pm v_{\mathrm{A}}+V$ (full line) and cut-off speed $\pm \omega_{\mathrm{II}} / k_{z}+V$ in a boundary layer of thickness 0.1 separating the incoming wave with wavenumbers $k_{z}=1 / \sqrt{2}=k_{y}$ and longitudinal phase speed $\omega / k_{z}=2$ from a region with $v_{\mathrm{Al}}=3$ and $V=4.6 . R$ and $R R$ indicate the amplitude and energy reflection coefficients. 


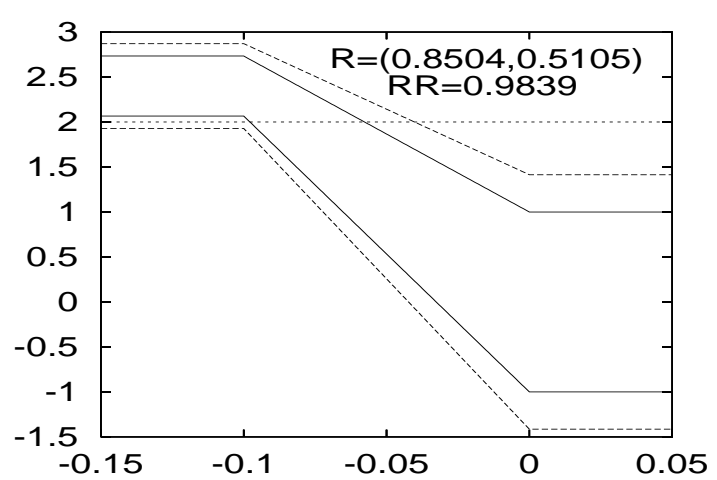

Fig. 4. Possible profiles for Alfvén speed $\pm v_{\mathrm{A}}+V$ (full line) and cut-off speed $\pm \omega_{\text {II }} / k_{z}+V$ in a boundary layer of thickness 0.1 separating the incoming wave with wavenumbers $k_{z}=1 / \sqrt{2}=$ $k_{y}$ and longitudinal phase speed $\omega / k_{z}=2$ from a region with $v_{\mathrm{Al}}=\frac{1}{3}$ and $V=2.4 . R$ and $R R$ indicate the amplitude and energy reflection coefficients.

seen from Fig. 7 the resonances have a strong absorbing effect and overcome the KH-type overreflection. However it was not easy to find parameters for which the resonant effects were strong enough to stabilize the non-resonant overreflection. This is closely related to the assumption of the "thin" boundary. As stated before the resonant effects vanish in the limit of $L \rightarrow 0$. It is thus not surprising that in case of a "thin" boundary layer the resonant effects are rather small as can be seen from the results. In formula (15) $D_{\mathrm{l}}$ and $D_{\mathrm{r}}$ depend inversely on $k$, whereas $D_{0}$ is roughly proportional to $L$ through the spatial derivative and independent of $k$. Therefore the resonant effects will be small as long as $L k \ll 1$. But they can be expected to be much larger when a "thick" boundary or slowly varying medium is considered. However the overreflecting and absorbing effects then can not be compared so clearly.

In order to obtain dominant resonant effects we have increased the angle between the wavevector and the magnetic field to $65^{\circ}$ ( $45^{\circ}$ before). This increases the effect of the resonances because $k_{y}$ acts as a coupling parameter. The larger $k_{y}$ the stronger the coupling with local Alfvén waves and thus the stronger the resonant effects. Analytically this is expressed by the $k_{y} / k_{z}$ factor in $D_{0}$. The change of the angle changes the cut-off frequencies as well and thus the $z$-component of the phase velocity had to be increased to 2.7 in order to have a propagating wave in the right medium.

Thus from these results we can conclude that stratification in the boundary layers is important, not only because it can introduce overreflection below the $\mathrm{KH}$ velocity threshold, but also because it can suppress overreflection above this threshold. Which is the case depends on the precise structure of the boundary layer and may possibly be different for incoming waves with different frequencies.

\section{Summary and discussion}

We have investigated the problem of reflection and transmission at a stratified boundary layer between two homogeneous plasmas. More specifically we addressed the influence of resonant wave coupling in the boundary layer. For sake of simplicity we assumed the boundary layer to be "thin". From the results we can conclude that stratification in the boundary layers can be important, not only because it can introduce overreflection below the KH velocity threshold, but also because it can suppress overreflection above this threshold. Which is the case depends on the precise structure of the boundary layer and may possibly be different for incoming waves with different frequencies. In general waves with large wavelengths are only slightly influenced by the resonant coupling effects in the boundary layer. While waves with small wavelengths are likely to be effected a lot more. More specifically resonant effects are very small if the wavevector of the incoming wave is oriented mainly along the magnetic field. However the resonant effects can become dominant even in the "thin" boundary approximation for oblique waves.

The relevance of this study is mainly due to the relationship between (over)reflection and the (over)stability of waveguide body modes. Waveguide body modes are body waves that are trapped between two boundaries, being reflected each time they reach a boundary. If the conditions are such that in the driven problem overreflection occurs, these waveguide modes grow. Thus in an eigenmode calculation the waveguide modes are overstable. For the stability of surface waves (or surface-type waveguide modes) these calculations are less relevant since they can not be related to a reflection and transmission problem since they are non-propagating. However similar profile dependency can be expected for the surface waves.

Recent studies did already show that the boundary layer and the occurring resonances may be important (for both surface and body waves) in the sense that it can lead to overstabilities for velocity shears below the KH threshold (Ryutova 1988; Hollweg et al. 1990; Tirry et al. 1998; Ruderman 1998; Andries et al. 2000; Andries \& Goossens 2001). However we have shown that these results can not be straightforwardly generalized. In fact these results are somewhat weakened by this study because it turns out that overstability was only obtained because the boundary layer profiles were taken in a way to favour overstability. However the correspondence between the model profiles and the profiles that occur in nature is at least uncertain. Thus realistic profiles could possibly produce very different results.

On the other hand this study shows that resonant coupling in the boundary layer is not only important because of the related destabilization mechanism but also because of its possibly stabilizing effect on the KH-istability. To avoid confusion, we repeat that we are talking about $\mathrm{KH}-$ instability of trapped waves and not about the classical surface wave KH-instability. However if similar effects are present for the surface waves, wave resonance could possibly provide an explanation for the stabilization of $\mathrm{KH}$ instability by the spreading of the boundary layer. If a boundary is KH-unstable the excited perturbations smear out the boundary layer, eventually reaching a state in 

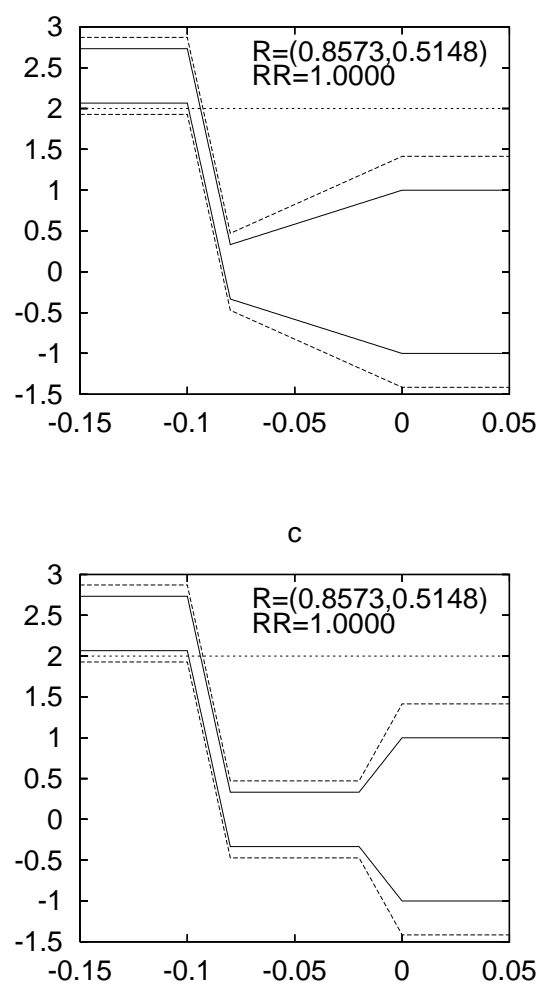

b

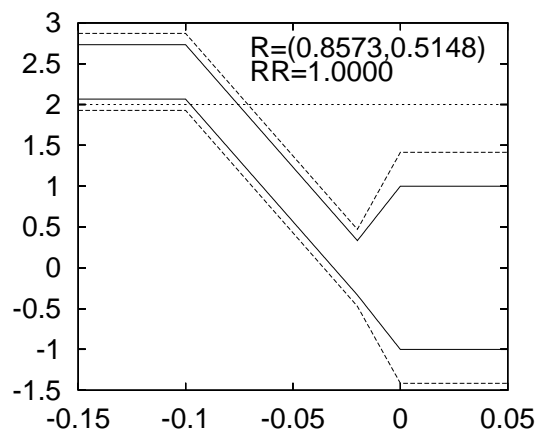

d

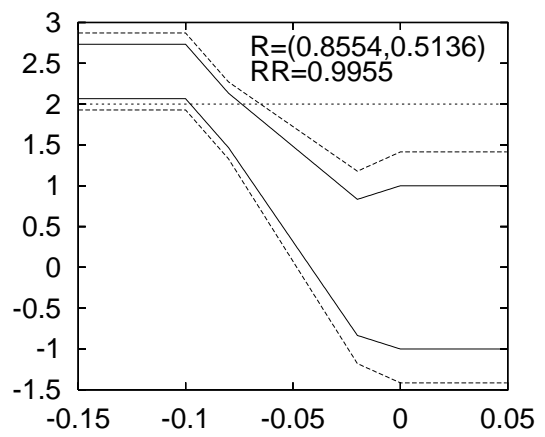

Fig. 5. Different possible profiles for Alfvén speed $\pm v_{\mathrm{A}}+V$ (full line) and cut-off speed $\pm \omega_{\mathrm{II}} / k_{z}+V$ in a boundary layer of thickness 0.1 separating the incoming wave with wavenumbers $k_{z}=1 / \sqrt{2}=k_{y}$ and longitudinal phase speed $\omega / k_{z}=2$ from a region with $v_{\mathrm{Al}}=\frac{1}{3}$ and $V=2.4$. $R$ and $R R$ indicate the amplitude and energy reflection coefficients.
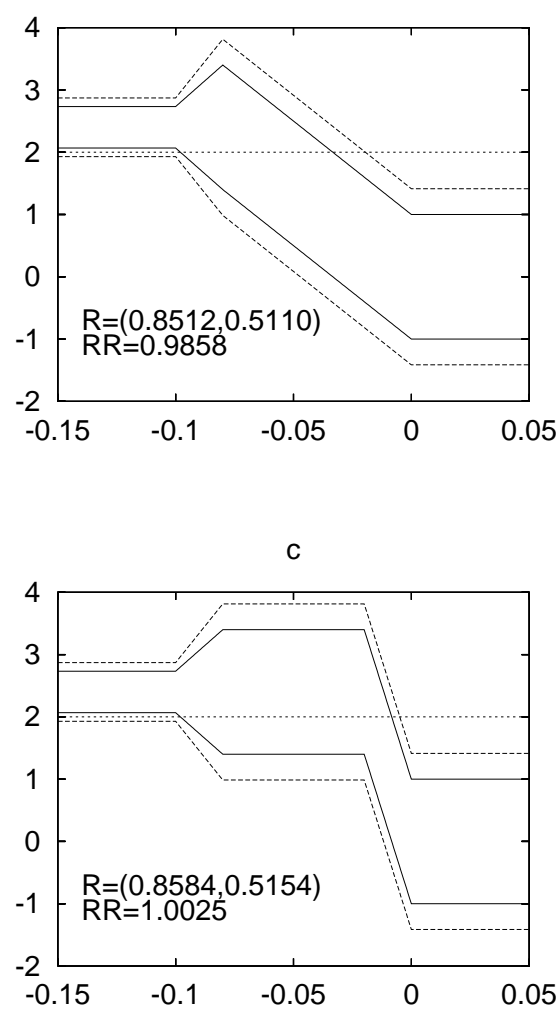

b

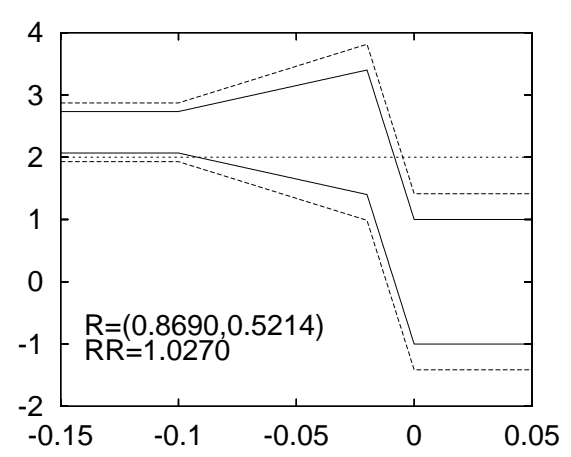

d

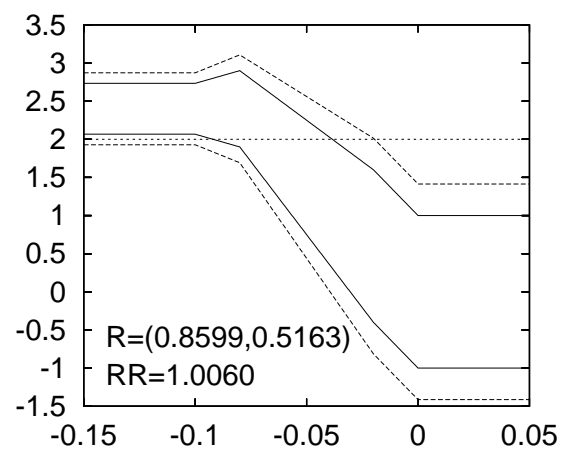

Fig. 6. Different possible profiles for Alfvén speed $\pm v_{\mathrm{A}}+V$ (full line) and cut-off speed $\pm \omega_{\mathrm{II}} / k_{z}+V$ in a boundary layer of thickness 0.1 separating the incoming wave with wavenumbers $k_{z}=1 / \sqrt{2}=k_{y}$ and longitudinal phase speed $\omega / k_{z}=2$ from a region with $v_{\mathrm{Al}}=\frac{1}{3}$ and $V=2.4 . R$ and $R R$ indicate the amplitude and energy reflection coefficients. 


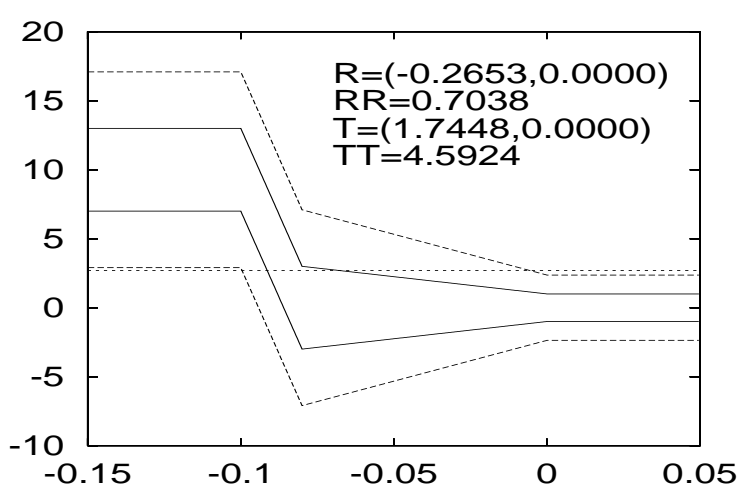

Fig. 7. Possible profiles for Alfvén speed $\pm v_{\mathrm{A}}+V$ (full line) and cut-off speed $\pm \omega_{\text {II }} / k_{z}+V$ in a boundary layer of thickness 0.1 separating the incoming wave with wavenumbers $k_{z}=1 / \sqrt{2}=$ $k_{y}$ and longitudinal phase speed $\omega / k_{z}=2$ from a region with $v_{\mathrm{Al}}=\frac{1}{3}$ and $V=2.4 . R, T, R R$, and $T T$ indicate the amplitude and energy reflection and transmission coefficients. Apparently these profiles result in resonant suppression of the KH-type overreflection.

which the boundary layer is stable with respect to $\mathrm{KH}-$ instability. A thicker boundary layer creates larger derivatives of the above mentioned profiles and thus enhanced influence of the resonant effects which could possibly be damping effects, thereby stabilizing the configuration.

We conclude that effects due to resonant coupling in a stratified boundary layer can not be neglected and can drastically influence the stability of the boundary layer in either way.
Acknowledgements. The authors are grateful to A. D. M. Walker for removing their "negative energy" wave blindfolds. J. A. would like to thank the University of Natal and the Flemish government (BIL97/49) for enabling his visit to the University of Natal, Durban.

\section{References}

Adam, J. A. 1978, Q. Jl. Mech. Appl. Math., 1, 77

Andries, J., Tirry, W. J., \& Goossens, M. 2000, ApJ, 531, 561

Andries, J., \& Goossens, M. 2001, A\&A, 368, 1083

Csík, Á. T., Čadež, V. M., \& Goossens, M. 1998, A\&A, 339, 215

Goossens, M., Hollweg, J. V., \& Sakurai, T. 1992, SPh, 138, 233

Goossens, M., Ruderman, M. S., \& Hollweg, J. V. 1995, SPh, 157,75

Hollweg, J. V., Yang, G., Čadež, V. M., \& Gaković, B. 1990, ApJ, 349, 335

Ruderman, M. S., \& Wright, A. N. 1998, JGR, 103, 26, 573

Ryutova, M. P. 1988, J. Exper. Theoret. Phys., 94, 138

Sakurai, T., Goossens, M., \& Hollweg, J. V. 1991, SPh, 133, 227

Sedlacek, Z. 1971, J. Plasma Phys., 6, 187

Tirry, W. J., \& Goossens, M. 1996, ApJ, 471, 501

Tirry, W. J., Čadež, V. M., Erdélyi, R., \& Goossens, M. 1998, A\&A, 332, 786

Walker, A. D. M. 2000, J. Plasma Phys., 63(3), 203

Yang, G., \& Hollweg, J. V. 1991, JGR, 96(13), 807 